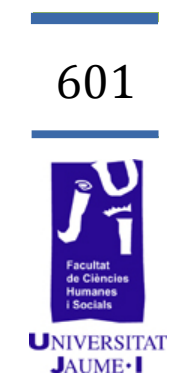

\title{
Las diferencias del diálogo entre gobierno-sociedad y entre empresa-sociedad. Revisión bibliográfica
}

Augusto Leiva Espinoza augustoleiva@hotmail.com 
Con el pasar del tiempo y la entrada de las nuevas generaciones, se han dado cambios de progreso social y de desarrollo dentro de las organizaciones. Existen líderes más jóvenes y se nota un leve incremento del género femenino en las posiciones de alta dirección de las organizaciones. Las estructuras organizacionales van realizando pequeñas modificaciones para tratar de adaptarse y responder con el contexto social y generacional del entorno. Sin embargo, la estructura central de las organizaciones aún conserva patrones relacionales y de comunicación establecidos desde sus inicios. Según las autoras Majken Schultz y Mary Jo Hatch, ${ }^{1}$ una correcta buena práctica dentro de las organizaciones es reforzar su cultura organizacional y valores corporativos a través de «la revisión de su identidad diferencial o corporativa para determinar la dirección estratégica» (Schultz y Hatch, 2008: 178). Por otro lado, autores como Oriol Iglesias y Nicholas Ind confirman la importancia de definir la identidad corporativa a través de la marca corporativa y enfatizan la importancia (de la identidad) en la empresa para marcar una perspectiva pasada, presente y futura que ayude a la toma de decisiones. ${ }^{2}$ Frente a este fenómeno organizativo surge la duda de saber si los valores iniciales de las empresas respondían con un contexto muy diferente del de ahora y esto se vea reflejado en la forma de comunicar que las organizaciones se encuentran efectuando en la actualidad. Es interesante saber si estos mensajes informativos de las organizaciones son recogidos o entendidos dependiendo de las diferentes generaciones como las de los Baby Boomers, Generación X y millennials, existentes en la sociedad. Partiendo de que el diálogo que mantiene la sociedad con las organizaciones públicas y privadas se sostiene con las relaciones dentro del contexto en el que se encuentran, es conveniente analizar si los mensajes que emiten las empresas y administraciones públicas encuentran una comprensión (o correcta interpretación) por parte de todas las generaciones que se encuentren con la capacidad suficiente para mantener un diálogo que contribuya con su desarrollo. La revisión bibliográfica de la información referente al tema de las diferencias generacionales en la comunicación institucional y pública para ver si existen brechas generacionales no actualizadas que se deben de analizar, arroja que las instituciones públicas y privadas responden generacionalmente con la relación que mantienen con sus grupos de interés. Esto significa que no solo se hablan de clientes o consumidores dentro de la sociedad, sino que implica una relación de interdependencia con otros públicos que son muy diversos y diferentes dependiendo del tipo de organización que se analice (pública o privada) y también del tipo de mercado en el que trabaje (sectores de banca, turismo, construcción, consumo, entre otros). Para el presente estudio, se ha logrado analizar la viabilidad de la comparación de los grupos de interés entre la empresa y la administración pública en los

1. Schultz y Hatch, Taking Brand Initiative: How Companies Can Align Strategy, Culture, and Identity.

2. Ind, y otras (ed.) (2013): Building Brands Together: Emergence And Outcomes of Co-Creation. 
empleados, la alta dirección y la relación con los organismos sin ánimo de lucro.

Palabras clave: stakeholders, generaciones, interpretación, comunicación, empresa-sociedad, gobierno-sociedad.

\section{Introducción}

Los estudios de la semiótica así como el desarrollo de la narrativa social son factores claves para la comunicación entre personas, grupos sociales, empresas y también instituciones. El común denominador son los sistemas de lenguaje (a través de diferentes formatos y estilos narrativos) que establecen una correcta comunicación en que la información que se emite es la que se recibe y viceversa. Sin embargo, existen componentes racionales y emocionales dentro del contexto comunicativo que afectan a la emisión y recepción de los mensajes. Esta particularidad se incrementa cuando se encuentran diferencias generacionales, que manejan el lenguaje desde contextos y condiciones totalmente diferentes entre sus interlocutores, que a su vez generan labores adicionales de interpretación.

Si llevamos esta premisa a la comunicación de instituciones públicas y privadas con la sociedad, encontraremos que cada actor social (empresa - administración pública) posee un estilo común o una subcultura organizacional (o corporativa) en la forma de comunicar sus mensajes. Estos rasgos distintivos de la organización vienen derivados de su identidad corporativa que la hace única en la forma de operar, contratar, abordar los problemas del mercado, comunicar productos y servicios, etc. Sin embargo, la organización es un conjunto de subgrupos de personas (divididos por áreas y jerarquías internas) que coexisten en un espacio común. Dentro de esta variedad humana que contiene la organización, los modelos jerárquicos establecen el orden interno y marcan la importancia y valor de los roles de cada persona dentro de este grupo organizativo. El principal líder de la empresa, o el que se sitúa en lo más alto de la jerarquía corporativa (CEO de la empresa o presidente del país), es el que marca la estrategia o la ruta a seguir para el beneficio de todos los que conforman la organización. El común denominador a lo largo de la historia es que este líder contenga la experiencia y conocimiento suficiente para lograr situarse en la punta más alta de la pirámide jerárquica y por ello la mayoría poseen edades promedio entre los cincuenta y los setenta años. También ha sido común a lo largo de la historia que estos líderes sean de género masculino.

Con el paso del tiempo y la entrada de las nuevas generaciones, se han sucedido cambios de progreso social y de desarrollo dentro de las organizaciones. Hay líderes más jóvenes y cada vez más se nota un incremento del género femenino en las posiciones de alta dirección de las 
organizaciones. Las estructuras organizacionales van realizando pequeñas modificaciones para tratar de adaptarse y responder con el contexto social y generacional del entorno. Existen cambios, traspasos de confianza, nuevas iniciativas, correcciones y propuestas que mejoran o alteran el orden anterior del equilibrio inicial. Una interesante reflexión del profesor Benavides que realiza sobre los nuevos espacios de diálogo entre las organizaciones y la sociedad ${ }^{3}$ donde sostiene que (Benavides, 2015: 12):

[...] en la actualidad conviven lógicas diferentes porque la percepción visual de la realidad es plural y debemos investigar y definir el suelo epistemológico que está detrás de los procesos de comunicación.

La estructura central de las organizaciones aún conserva patrones relacionales y de comunicación establecidos desde sus inicios. Una correcta buena práctica dentro de las organizaciones es reforzar su cultura organizacional y valores corporativos a través de la revisión de su identidad diferencial o corporativa (Schultz y Hatch) para determinar la dirección estratégica y marcar una perspectiva pasada, presente y futura que ayude a la toma de decisiones (Ind e Iglesias). Ind ha desarrollado en otra investigación titulada Living the Brand: How to Transform Every Member of Your Organization into Brand Champion en el año 2007 que la cultura corporativa se ve reflejada en la forma de comunicar y de actuar que las organizaciones se encuentran efectuando en la actualidad. ${ }^{4} \mathrm{El}$ estudio de Edelman en 2012 que mide la confianza en las empresas e instituciones en el ámbito mundial, llamado Trust Barometer, muestra que, de la lista de los temas importantes que valoraba la sociedad en el año 2012, las instituciones presentaban temas de poco interés para las personas que los ubicaban en los últimos puestos de la lista de temas relevantes. ${ }^{5}$ Aquello, además de reflejar este desnivel de atención en los mensajes con las necesidades sociales de las organizaciones hacia la sociedad, también enfatiza una lenta reacción de las organizaciones ante los cambios coyunturales (provocados por las nuevas generaciones) tanto para abordar temas sociales sensibles como para poder responder 0 dialogar en el mismo nivel y con la misma empatía y entendimiento de las nuevas necesidades generacionales.

3 Benavides, El nuevo diálogo social: organizaciones, públicos y ciudadanos, p.12, 2015.

4 Ind, N. (2007): Living the Brand: How to Transform Every Member of Your Organization into a Brand Champion.

5 Edelman (2012): Trust Barometer. 


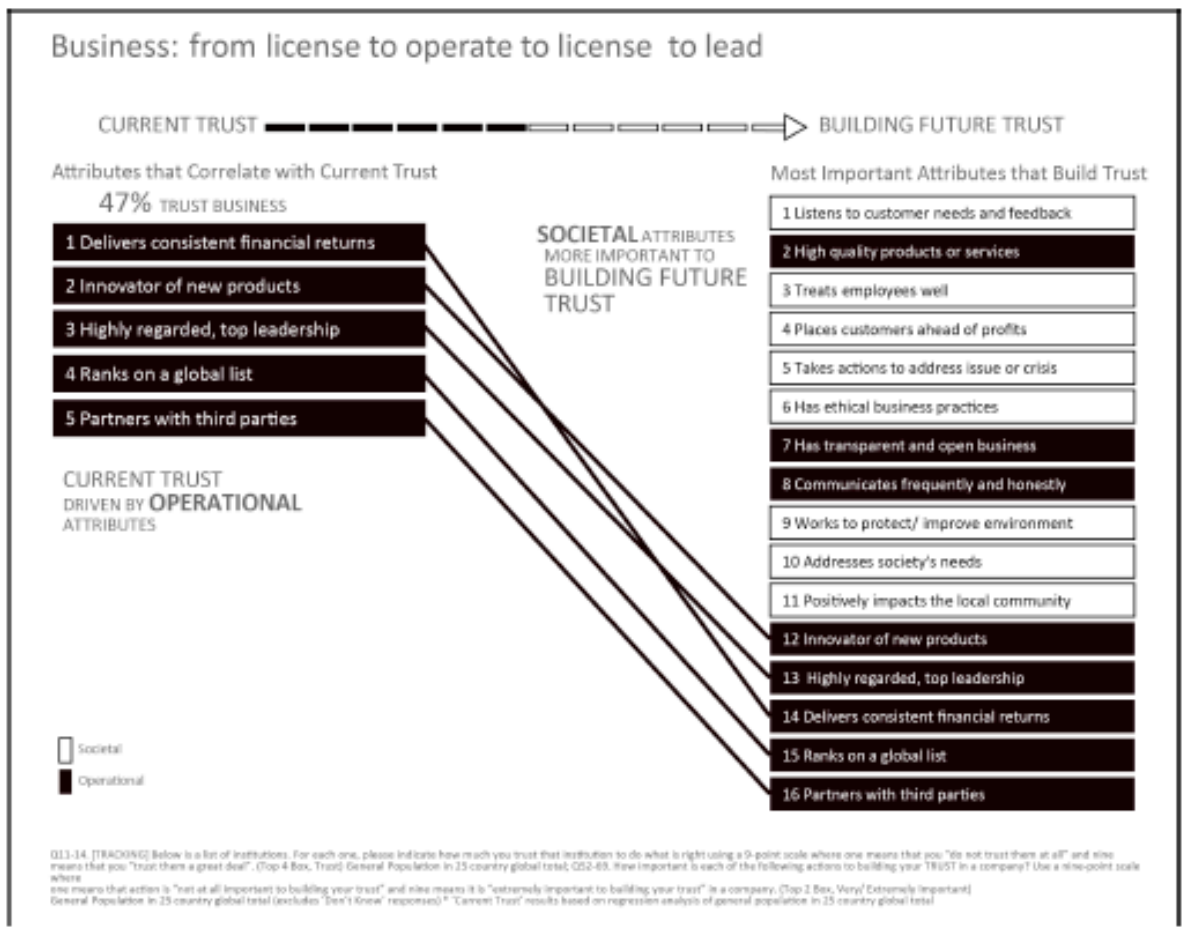

Figura 1. Business: from license to operate to license to lead. Edelman, Trust Barometer, 2012

Una lectura inicial o primera conclusión sería que los cambios generacionales van más rápido que las estructuras organizativas y ello se puede comprobar en la forma en la que, tanto empresas como instituciones públicas, emiten la información y cuánto tarda esta en ser procesada, interpretada y comprendida en la sociedad en sus diferentes generaciones.

Parece conveniente que se analicen las diferencias de comprensión e interpretación de los mensajes que existen entre las organizaciones y las diferentes generaciones dentro de la sociedad. Una primera hipótesis a comprobar en futuras investigaciones cualitativas y cuantitativas es la existencia de una mayor comprensión de los mensajes que las organizaciones públicas y privadas con las generaciones nacidas entre los años 1940 y 1950 debido a que el desarrollo de las estructuras organizativas tuvieron un desarrollo después de la Segunda Guerra Mundial y tanto las empresas como las administraciones públicas podían responder con los mismos niveles de lenguaje y comunicación que frente a la sociedad de esa época.

Si las empresas y administraciones públicas se encuentran emitiendo mensajes informativos solo comprensibles para un grupo determinado de la sociedad y el aspecto generacional tiene una influencia en esta diferencia o sesgo, son dos aspectos centrales de la investigación ya que genera nuevas incógnitas y líneas de investigación para demostrar cuál de los dos actores (empresa o administración pública) posee menos problemas de interpretación por parte de la sociedad y cuál se encuentra muy distante de comprensión por parte de las nuevas generaciones. Es 
conveniente resaltar que los problemas de interpretación o compresión de los mensajes contribuyen o refuerzan la percepción negativa o positiva que la sociedad tiene sobre la empresa y la administración pública y aquello establece las condiciones suficientes para determinar su legitimidad y diferenciación. ${ }^{6}$

Por lo tanto, partiendo de que el diálogo que mantiene la sociedad con las organizaciones públicas y privadas sostiene las relaciones dentro del contexto, la revisión bibliográfica sirve a la investigación para analizar si los mensajes que las empresas y administraciones públicas encuentran una buena comprensión o correcta interpretación por parte de las todas generaciones que se encuentren con la capacidad suficiente para mantener un diálogo que contribuya con su desarrollo, en especial para las nuevas generaciones denominadas millennials que buscan introducirse al mercado laboral y buscan ofertas laborales de las organizaciones públicas y privadas en todas sus variantes comunicativas.

\section{Objetivos}

Llegar a determinar si existe un compendio bibliográfico que nos permita conocer el estado de la cuestión sobre la forma en que las instituciones públicas y organizaciones privadas dialogan con las nuevas generaciones mejor entendidas como millennials.

Encontrar una relación entre los mensajes de las organizaciones públicas y privadas con las generaciones nacidas entre los años 1980 y 1995 que nos permita ver las dificultades de interpretación y entendimiento de las organizaciones con las generaciones.

Encontrar similitudes de grupos de personas con los que ambas organizaciones se relacionan para encontrar una viabilidad comparativa que se pueda analizar en investigaciones futuras.

\section{Material y método}

Para el cumplimiento de los objetivos trazados, se ha realizado un acopio de materiales de fuentes primarias y secundarias (vídeos, audios, libros, artículos, entrevistas no propias, estudios, lecturas monográficas, etc.) en relación con la comunicación de organizaciones públicas y privadas; con la gestión de la identidad, comunicación y marca corporativa; con los problemas de comunicación e interpretación por parte de los públicos de las organizaciones, y con los grupos de interés de las empresas e instituciones públicas. Dichos materiales serán nacionales e internacionales para llegar a tener una bibliografía vasta sobre los temas tratados. Posterior al acopio de materiales para la investigación, se procederá con la redacción del trabajo y la extracción de conclusiones.

6. Carreras, Alloza y Carreras (2013): Reputación Corporativa. 
De la revisión bibliográfica focalizada en la comunicación de las organizaciones públicas y privadas con la generación millennial, la diferencia de entendimiento y comprensión de los mensajes que emiten tanto la empresa como la administración pública hacia las personas que buscan iniciarse en el mundo laboral, se encuentra condicionada por el rango generacional y de antigüedad de las organizaciones. Esta primera conclusión se logra gracias al análisis de la relación de mensajes de las organizaciones con su comprensión generacional. Para realizar este análisis, se ha partido de tres tipos de organizaciones divididas por su antigüedad en la sociedad que se contrastarán con tres tipos de generaciones divididas por los rangos de edades comprendidas entre los 20 a 24 años, 25 a 29 años y 30 a 35 años, respectivamente. La aplicación de ambos ejes (organizaciones vs. generaciones) se puede apreciar en la Tabla 1:

\section{Relación de mensajes de las organizaciones con su comprensión generacional}

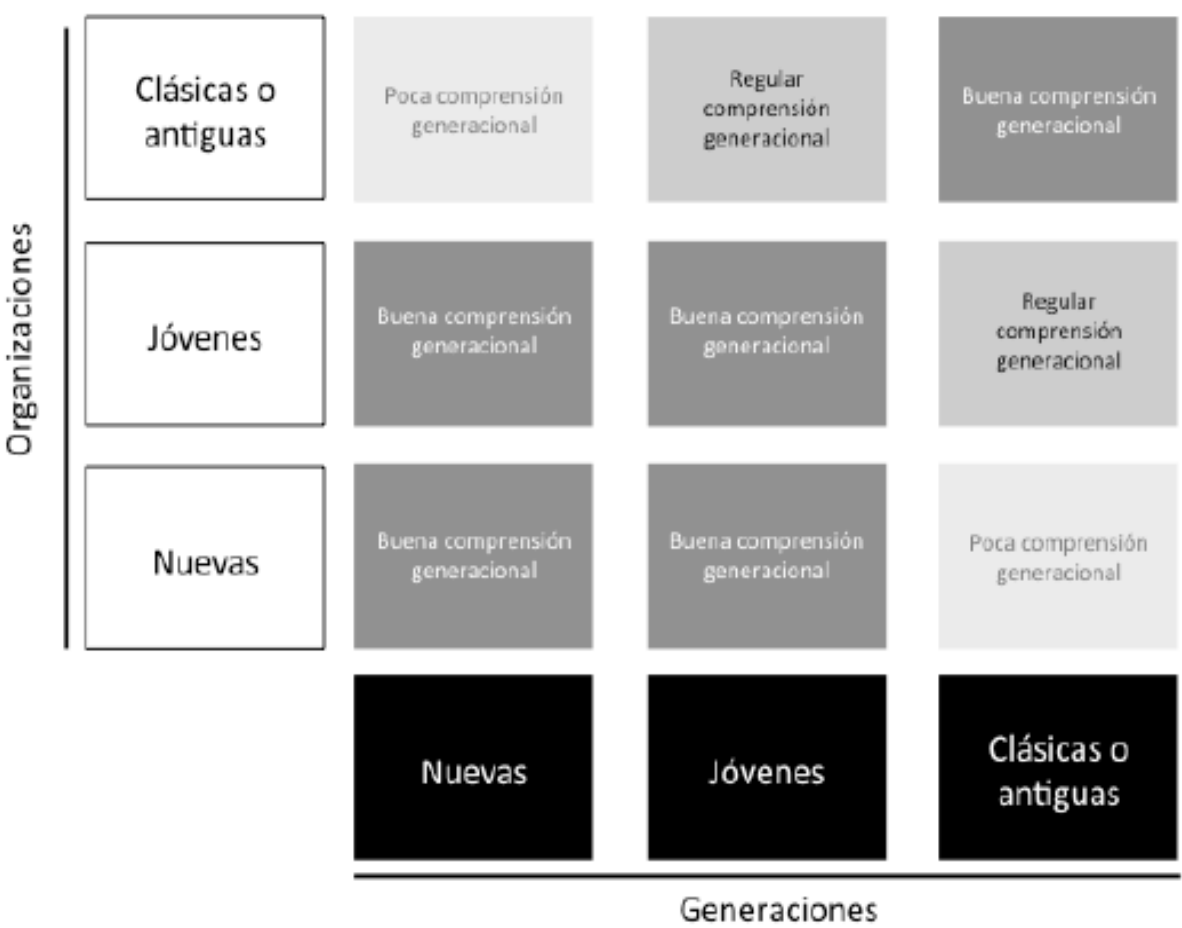

Tabla 1. Hipótesis de la relación de mensajes de las organizaciones con su comprensión generacional. Elaboración propia.

De la Tabla 1 se pueden extraer las siguiente conclusiones:

Existe una relación directa de la antigüedad de las organizaciones con su símil generacional. Es decir, una organización con más años dentro de la sociedad ha sabido comprender a las generaciones más antiguas, mientras que las organizaciones jóvenes y nuevas encuentran comprensión y entendimiento con las dos generaciones más jóvenes de las tres analizadas. 
Existe una generación puente entre las generaciones nuevas y las antiguas y es la que se encuentran en mitad de ambas debido a que pueden comprender los argumentos para la comprensión entre ambas generaciones.

Tanto las generaciones nuevas como antiguas poseen pocas líneas de entendimiento entre ambas cuando se trata de comunicación organizacional. Ello hace que una de las diferencias entre organizaciones nuevas y clásicas sea su rápida capacidad de respuesta y adaptación al cambio que tienen las primeras versus las segundas.

Las generaciones más antiguas logran entender las estructuras organizativas sociales y, por ende, su comunicación. Sin embargo, aquello no implica que las nuevas organizaciones logren cambiar las bases estructurales sociales con el paso de los años. Iriso y Rubio sostienen que son las organizaciones públicas las que deberían de llegar a realizar intentos de acercamiento social para su mejor comprensión en cuanto a necesidades e intercambio de propuestas; ${ }^{7}$ aunque también plantean problemas de entendimiento e interpretación de los mensajes debido al paradigma que maneja cada ciudadano por separado y en conjunto al no lograr diferenciar organizaciones públicas de la administración (Rubio e Iriso, 2010: 27):

Podríamos decir que vivimos en una jungla comunicativa en la que se entremezclan los intereses de todos los sectores público y privado [...] La audiencia recibe la información de una manera selectiva. Hay cosas que pueden no interesar a unos y sí a otros.

Durante la revisión bibliográfica se han encontrado muchos criterios aún más abiertos para la investigación, estos arrojaban criterios generacionales y también de grupos de interés que no se ajustaban con el objeto de la investigación pero que sin embargo ha servido para comprobar conceptos generacionales y culturales que servirán de contraste para una futura investigación cualitativa derivada de esta inicial. Se ha centrado el objeto de estudio en la revisión de la bibliográfica de las investigaciones y publicaciones que aborden las generaciones nacidas entre 1980 y 1995. Con las investigaciones que aborden este espectro de personas se puede elaborar un análisis más enfocado partiendo de las necesidades de inclusión y preparación para el mundo laboral que posee este grupo de personas, así como el análisis de los mensajes de la Administración Pública emite en ello sobre empleabilidad y también los mensajes de las empresas en la búsqueda de puestos de trabajo.

Un primer hallazgo que se encuentra durante la revisión bibliográfica de la investigación, es la escasa existencia de material de investigación académica de los espacios comunicativos donde no se pueda realizar una comprobación simultánea tanto de la Administración Pública como de las organizaciones privadas o empresas, circunscrita en la generación

7. Rubio e Iriso, (2010): Comunicar para compartir: Un viaje hacia una mejor comunicación pública. 
analizada y que aporte una reciente novedad al objeto. Esto se debe a que el tema de los millennials es novedoso para el análisis y veremos nuevos hallazgos sobre esta generación y su relación con las organizaciones en los años siguientes. Frente a esta premisa, se recurrió a la teoría de los grupos de interés, concepto introducido en 1984 por Edward Freeman en su obra Strategic Management: A Stakeholder Approach en el que afirma que existen agrupaciones de personas vinculadas e interesadas en las organizaciones. ${ }^{8} \mathrm{El}$ concepto de los stakeholder o grupos de interés ha dado un cambio importante en la gestión empresarial ya que, a finales de la década de los ochenta, los directivos y gestores comienzan a interesarse en preguntar por las necesidades y opiniones de otros grupos de personas diferentes de los clientes o consumidores.

Si bien la teoría de los grupos de interés de Freeman enfatiza la relación de los grupos de personas con la organización, para la presente investigación se ha ajustado esta teoría en que los grupos de interés sean los mismos tanto para la empresa como para la Administración Pública. Producto de este trabajo de homologación de grupos de interés en que se puedan encontrar puntos de relación de las dos organizaciones, se ha desarrollado una comparabilidad entre ambas entidades de donde se podrá extraer las líneas de trabajo para la presente investigación. De esta manera se listarían los diferentes los grupos de interés de la empresas y de la Administración Pública y se describiría cuáles son los grupos de interés en ambas organizaciones $\mathrm{y}$ si es viable $u$ homologable la comparabilidad de la investigación. Para el análisis de los grupos de interés y su comparabilidad entre las organizaciones públicas y privadas, se tomarán en consideración los nueve grupos de interés considerados por Freeman, a saber: clientes, consumidores, empleados, administración pública, accionistas, proveedores, alta dirección, competidores y organizaciones sin ánimo de lucro u ONG. El desarrollo se puede observar en la Tabla 2: 
Viabilidad de la comparación de Grupos de Interés entre Empresa y Administración Pública

\begin{tabular}{|c|c|c|c|}
\hline Grupas de interés & Empresa & Administración Pública & $\begin{array}{l}\text { Mabilidad de } \\
\text { comparación }\end{array}$ \\
\hline Clientes & Personas que compran productos o servicios & $\begin{array}{l}\text { No diene - los ciuda danos pueden llegara a } \\
\text { acercarse a esta definicion }\end{array}$ & $\begin{array}{l}\text { No se puede } \\
\text { comparar }\end{array}$ \\
\hline Consumidores & $\begin{array}{l}\text { Personas que consumen los productos y servicios } \\
\text { que se comercializa }\end{array}$ & $\begin{array}{l}\text { Personas que consumen los servicios } \\
\text { públicos - los ciudadanos }\end{array}$ & $\begin{array}{l}\text { No se puede } \\
\text { comparar }\end{array}$ \\
\hline Empleados & $\begin{array}{l}\text { Personas que prestan servicios retribuidos y } \\
\text { subcrdinados }\end{array}$ & $\begin{array}{l}\text { Personas pue prestan servicios retribuidos } \\
\text { y subordinados }\end{array}$ & $\begin{array}{l}\text { Se puede } \\
\text { comparar }\end{array}$ \\
\hline $\begin{array}{l}\text { Administración } \\
\text { Pública }\end{array}$ & $\begin{array}{l}\text { Grupo de personas y organismos que dirigen una } \\
\text { división político-administrativa en la sociedad }\end{array}$ & Son ellos mismos. & $\begin{array}{l}\text { No se puede } \\
\text { comparar }\end{array}$ \\
\hline Accionistas & $\begin{array}{l}\text { Grupos de personas que invierten un capital } \\
\text { economico que pertenecen a una sociedad } \\
\text { financiera, comercial o industrial. }\end{array}$ & $\begin{array}{l}\text { No diene - los ciudadanos pueden llegara a } \\
\text { acercarse a esta definicion a travts del } \\
\text { pago de impuestos }\end{array}$ & $\begin{array}{l}\text { No se puede } \\
\text { comparar }\end{array}$ \\
\hline Proveedores & $\begin{array}{l}\text { Grupos de empresas que se dedican a abastecer } \\
\text { de productos o servicios necesarios a una } \\
\text { empresa. }\end{array}$ & $\begin{array}{l}\text { Grupos de empresas que se dedican a } \\
\text { abastecer de productos o servicios } \\
\text { necesarios a una empresa. }\end{array}$ & $\begin{array}{l}\text { Se puede } \\
\text { comparar }\end{array}$ \\
\hline Alta dirucción & $\begin{array}{l}\text { Personas de una empresa con a ltos cargos y de } \\
\text { elevada responsabilidad que participan en la } \\
\text { toma de decisiones estratégicas }\end{array}$ & $\begin{array}{l}\text { Personas de la administración con altos } \\
\text { cargos y de elevacia responsabilidad que } \\
\text { participan en la toma de decisiones } \\
\text { estratégicas }\end{array}$ & $\begin{array}{l}\text { Se puede } \\
\text { comparar }\end{array}$ \\
\hline Competidores & $\begin{array}{l}\text { Empresas que poseen los mismos objetuvos } \\
\text { comerciales con productos y servicios sim lares }\end{array}$ & No tene & $\begin{array}{l}\text { No se puede } \\
\text { comparar }\end{array}$ \\
\hline $\begin{array}{l}\text { Organizamos sin } \\
\text { ánimo de lucro - ONG }\end{array}$ & $\begin{array}{l}\text { Agrupaciones de iniciativa social y fines } \\
\text { humanitarios, que son independiantes de la } \\
\text { administración pública y que no tienen afán } \\
\text { lucrativa. }\end{array}$ & $\begin{array}{l}\text { Agrupaciones de iniocativa social y fines } \\
\text { humanitafios, que son independientes de } \\
\text { Ia administración pública y que no tienen } \\
\text { atán lucrativa. }\end{array}$ & $\begin{array}{l}\text { Se puede } \\
\text { comparar }\end{array}$ \\
\hline
\end{tabular}

Tabla 2. Viabilidad de la comparación de grupos de interés entre empresa y Administración Pública. Elaboración Propia

De la tabla se pueden extraer las siguientes conclusiones: no todos los grupos de interés de la empresa son comparables con los grupos de interés de la Administración Pública debido a que no poseen la misma naturaleza de sus funciones o de su sostenibilidad, aquello hace que ambas organizaciones gestionen de forma muy diferente la relación que mantienen con sus grupos de interés.

Los grupos de interés que podrían ser comparables para la investigación -que en este caso serían los empleados, los proveedores, y las organizaciones sin ánimo lucrativo- poseen variantes en las que, de momento, no es necesario profundizar pero que servirán para determinar las nuevas líneas de investigación que pueden desprenderse de este trabajo.

Otra interesante conclusión es que, tal como se vio en la Tabla 1, existen cruces generacionales entre iguales y entre las generaciones intermedias con las otros dos restantes, e incluso dentro de la misma organización con sus grupos de interés. Por ejemplo, para el caso de proveedores, si bien ambas organizaciones tienen la necesidad de un abastecimiento de recursos materiales, los requerimientos que derivan en negocios de empresa a empresa (o Business to Business) en las que la relación se hace a través de comerciales o agentes que se encuentran en las generaciones Baby Boomers o Generación $X$ debido a la empatía generacional que existe entre las empresas proveedoras con sus clientes (es decir que la edad de los agentes comerciales se equilibra con la edad de sus clientes para reforzar una mayor empatía y facilitar la venta). Otro caso similar se puede ver en la relación con los organismos sin fines de 
lucro debido a su composición mixta. Si bien las ONG necesitan tanto de las empresas (a través de sus voluntariados corporativos, la filantropía y la cooperación para el desarrollo) como de las administraciones publicas (a través de las subvenciones para proyectos en campo o su grado de influencia facilitando información técnica y social), la gama generacional se sigue estructurando a través de la jerarquía clásica de los años de experiencia y edad, y también al mismo factor de empatía frente a las relaciones, es decir, rango de edad similar entre interlocutores para reforzar las negociaciones (Tabla 1). Se tendrían que analizar elementos en los que ambos grupos se encuentren sin condicionantes y que la comunicación sea claramente visible en los canales comunicativos de la información.

El grupo de interés que guarda cierta comparabilidad entre empresa y Administración Pública son los empleados ya que estos obedecen a unas directrices de la organización y presentan un rango de edades muy variado para poder elegir en la investigación. Sin embargo, los empleados poseen la misma jerarquía de experiencia laboral en su estructura organizativa. Otra diferencia resaltante entre los empleados de las empresas en comparación con los empleados de la Administración Pública es que los primeros obedecen a un sueldo y están supeditados a cumplir con los resultados a costa de su salario o su puesto de trabajo. Muy por el contrario, los empleados de la Administración Pública provienen de las oposiciones que concede el Estado para poder trabajar dentro de los organismos de la Administración Pública. Es decir que su sueldo no está condicionado con los objetivos que la Administración Pública presente. Por ello, a los empleados de esta se les llama funcionarios y a los de la empresa, empleados. Sin embargo la empleabilidad posee intereses comunes en las personas de la sociedad que desean empezar en el mundo laboral. Tanto empresa como Administración Pública ofrecen oportunidades de empleo y becas formativas para optimizar el trabajo, para personas que se encuentran en un rango de edad que interesa a la investigación. Es, por tanto, la empleabilidad y las becas, una oportunidad para iniciar la investigación debido a que tanto empresa como administración pública emiten mensajes de acuerdo con su cultura organizacional que pueden ser o no entendidos por las personas interesadas en las ofertas laborales o de becas para su formación profesional.

\section{Discusión y conclusiones}

La revisión bibliográfica de la información referente al tema de las diferencias generacionales en la comunicación institucional para ver si existen brechas generacionales no actualizadas que se deben analizar arroja que las instituciones públicas y privadas responden generacionalmente con la relación que mantienen con sus grupos de interés. Aquello se debe a que cada actor social (empresa o 
administración pública) posee un estilo común o una subcultura organizacional (o corporativa) en la forma de comunicar sus mensajes. Otro factor de la relación es la coincidencia temporal, es decir, que las organizaciones que poseen edades similares a las generaciones a las que se dirigen poseen mejor comprensión frente a generaciones más jóvenes; así como las organizaciones jóvenes saben dejarse entender e interpretar con generaciones jóvenes o nuevas.

Se ha logrado analizar la viabilidad de la comparación de los grupos de interés entre la empresa y la Administración Pública utilizando la teoría de Freeman en tres grupos de interés que son los empleados, la alta dirección y la relación con los organismos sin ánimo de lucro. Para la presente investigación se han eliminado las relaciones generacionales similares para tratar de encontrar un contraste virtuoso y no de necesidad estructural o económica; producto de ello se han eliminado los grupos de interés de las organizaciones sin ánimo lucrativo y la alta dirección. Del grupo de interés de empleados, se ha separado a las personas que tienen una empleabilidad de las que recién buscan entrar en el mundo laboral. Se realizó esta separación para poner en el mismo nivel a empresa y Administración Pública en la oferta laboral en la sociedad ya que se dirigen al mismo público (gente de 20 a 35 años).

El presente trabajo de revisión bibliográfica ha arrojado dos conclusiones teóricas que valdría seguir ampliando y enriqueciendo con líneas de investigación cualitativas y cuantitativas futuras que darían más solvencia a lo trabajado hasta el momento.

VII. Bibliografía

Aaker, D. A.; E. Joachimsthaler (2009): Brand Leadership, The Free Press.

ARGENTI, P. (2014): Comunicación Estratégica y su contribución con la reputación corporativa, LID Editorial.

BovalRD, T., y E. Löffler (2003): Public Management and Governance, Rouledge.

CAPRIOTTI, P. (1992): La imagen de empresa: estrategia para una comunicación integrada. El Ateneo.

Carreras, E. y otros (ed.) (2013): Reputación Corporativa, LID Editorial.

CARroll, C. E. (2013): The Handbook of Communication and Corporate Reputation, Wiley-Blackwell.

Fombrun, C. y C. B. M. Van Riel, (2004): Fame \& Fortune, Prentice Hall.

Freeman, E. (1984): Strategic Management: A Stakeholder Approach, Cambridge University Press.

HATCH, M. J., y M. SCHULTZ (2008): Taking Brand Initiative: How Companies Can Align Strategy, Culture, and Identity Through Corporate Branding, Jossey Bass. 
IND, N. (2007): Living the Brand: How to Transform Every Member of Your Organization into a Brand Champion, 3rd Edition, Kogan Page.

- (2012): Brand Together, Kogan Page.

Marín, F. (2009): Comunicación de crisis, Colección Acción Empresarial. LID Editorial.

MontaÑÉs DuATO, P. (2011): ¿Aquí quién manda? Levantando el mapa del poder en las organizaciones del siglo XXI, Biblioteca Altrán, Vol. 2 Pearson Educación.

RIES, A. y L. RIES (2005): El origen de las marcas. Empresa Activa.

RUBIO, L. y R. IRISO (2010): Comunicar para compartir: Un viaje hacia una mejor comunicación pública. Sinergia Papers.

Schmiтt, B. H. (2003): Customer Experience Management, John Wiley \& Sons Inc.

Sonnenfeld, A. (2011): Liderazgo ético: La sabiduría de decidir bien. Segunda Edición, Encuentro.

Stengel, J. (2011): Grow: How Ideals Power Growth and Profit at the World's Greatest Companies, Crown Business.

TrujlLlo, E. (2013): La sociedad que no quería ser anónima. Colección Acción Empresarial. LID Editorial.

Villafañe, J. (2004): La gestión profesional de la imagen corporativa. Pirámide.

- Artículos:

Aaker, J.; S. Foumier; S. Brasel; S. Adam (2008): «When Good Brands do Bad», Center for Responsible Business, UC Berkeley, Working Paper Series, Journal Of Consumer Research, Inc., Vol. 31.

Alloza, Á; S. Conley; F. Prado; J. Farfan y R. Espantaleon (2004): «Creating the BBVA Experience: Beyond Tradictional Brand Management", Corporate Reputation Review, Vol. 7, núm. 1, p. 66-81.

BALMER, John M.T. (2001): "Corporate Identity, Corporate Branding and Corporate Marketing: Seeing Through The Fog», Bradford School of Management, European Journal of Marketing, Vol. 35 No. 3/4, p. 248291.

BIVAINIENE, L. (2010): "Brand Life Cycle: Theoretical Discourses», Siauliai University, Economics and Management, p. 15.

BowkeR, D. (2009): "The Public Relations Perspective On Branding», Brands and Branding, 2nd Edition, Cap. 10, p. 146-156.

Dauvergne, P. y J. Lister (2011): «Big Brand Sustainability: Governance Prospects and Environmental Limits», Liu Institute for Global Issues, University of British Columbia, The Global Environmental Change.

De Chernatony, L. (2001): «Would a Brand Smell Any Sweeter by a 
Corporate Name?», Birmingham University Business School.

GaINES-Ross, L. (2010): «Reputation Warfare, Spotlight on Social Media and The New Rules of Branding», Hardvard Business Review, diciembre, p. 70-76.

Harris, F. y L. De Chernatony (2001): «Corporate Branding and Corporate Brand Performance», Open University Business School and The Birmingham Business School, European Journal of Marketing, Vol. 35 No. 3/4, p. 441-456.

HATCH, M. J. y M. SCHULTZ (2003): «Bringing the corporation into corporate branding", Mclntire School of Commerce, University of Virginia, Charlottesville, Virginia, USA, y Copenhagen Business School, Copenhagen, Denmark, European Journal of Marketing, Vol. 37 No. 7/8, p. 1041-1064.

- (2010): «Toward a Theory of Brand Co-Creation with Implications for Brand Governance», Brand Management, p. 1-15.

Iglesias, O.; A. Sauquet; y J. Montaña (2011): "The Role of Corporate Culture in Relationship Marketing», ESADE, Universitat Ramon Llull, European Journal of Marketing, Vol. 45 núm. 4, p. 631-650.

IND, N. (2007): «Branding Governance», Brandmanager, núm. 2, p. 30-37.

IND, N.; O. IGLESIAS; M. SCHULTZ (2013): «Building Brands Together: Emergence And Outcomes of Co-Creation», California Management Review, Vol. 55, núm. 3 Spring.

LANE KelleR, K. (2010): "The New Branding Imperatives: Insights for the New Marketing Realities», Marketing Science Institute.

SCHULTZ, M. (2011): «Relationships Between Culture and Institutions: New Interdependencies in a Global World?», Journal of Management Inquiry 2012 21: 102 originally published online 28 December 2011.

URDE, M.; S. A. GReyser y J. M. T. Balmer (2007): «Corporate Brands with a Heritage», Working Paper Series, núm. 07/18, July.

- Estudios, publicaciones y memorias académicas:

Arthur W. Page Society (2012): «Building Belief: A New Model For Activating Corporate Character And Authentic Advocacy». Arthur W. Page Society.

Bajo, A. y N. Villagra García (2012): "Los desafíos de la empresa ante la sostenibilidad: Diagnóstico, reflexiones y propuestas». Memoria Académica Curso 2011-2012. Reflexiones Comillas «Economía y Empresa». Universidad Pontificia de Madrid.

Corporate Excellence - Centre For Reputation Leadership (2012): «Asuntos Públicos: Marco conceptual y modelo de gestión. Ranking Global Issues 2012». Corporate Excellence - Centre for Reputation Leadership y Aula de Liderazgo Público de la Universidad Pontificia ICADE Comillas, 2012. 
Edelman (2012, 2013, 2014 y 2015): Trust Barometer, Eshuis, J. \& E. H. KLIJN, (2012): «Branding in Governance and Public Management». Routledge.

ESIC (2011): AdResearch ESIC: Revista Internacional de Investigación en Comunicación, núm. 3, primer semestre, enero-junio.

Foro Académico de Investigación en Comunicación (2015): El nuevo diálogo social: organizaciones, públicos y ciudadanos. 